\title{
Publisher Correction: Miniature two-photon microscopy for enlarged field-of-view, multi-plane and long-term brain imaging
}

Weijian Zong, Runlong Wu D, Shiyuan Chen, Junjie Wu, Hanbin Wang, Zhe Zhao, Guoqing Chen, Rui Tu, Danlei Wu, Yanhui Hu, Yangyang Xu, Yao Wang, Zhuoli Duan, Haitao Wu (D), Yunfeng Zhang (D), Jue Zhang (D), Aimin Wang (iD, Liangyi Chen (iD) and Heping Cheng (iD)

Correction to: Nature Methods https://doi.org/10.1038/s41592-020-01024-Z, published online 6 January 2021.

In the version of this article initially published, the accompanying Nature Research Reporting Summary was for a different paper. In addition, ref. 6 was cited at the end of the second sentence of main text; the correct citation is ref. 10. The errors have been corrected in the PDF and HTML versions of the article.

Published online: 22 January 2021

https://doi.org/10.1038/s41592-021-01066-x

(c) The Author(s), under exclusive licence to Springer Nature America, Inc. 2021 